\title{
Inhomogeneous Injection in III-Nitride Diode Structures with Deep Quantum Wells
}

\author{
Mikhail V. Kisin and Hussein S. El-Ghoroury \\ Ostendo Technologies Inc. \\ Carlsbad, CA, USA
}

\begin{abstract}
Excessive depth of optically active quantum wells (QWs) in III-nitride light emitters of visible range is one of the main causes of inhomogeneous carrier injection in multiplequantum-well (MQW) device active region. Deeper QWs imply stronger inhomogeneity of carrier distribution across the active region and support large residual charges of marginally located N-side and P-side QWs. Drift-diffusion transport approximation underestimates the minority carrier supply to marginal QWs which results in higher degree of injection inhomogeneity in transport simulation. We show that electron ballistic overshoot of narrow III-N QWs notably improves the carrier transport in MQW active region but has controversial effect on $Q W$ population uniformity.
\end{abstract}

Keywords-light emitting diodes; quantum wells; carrier injection; semiconductor device modeling; numerical simulations.
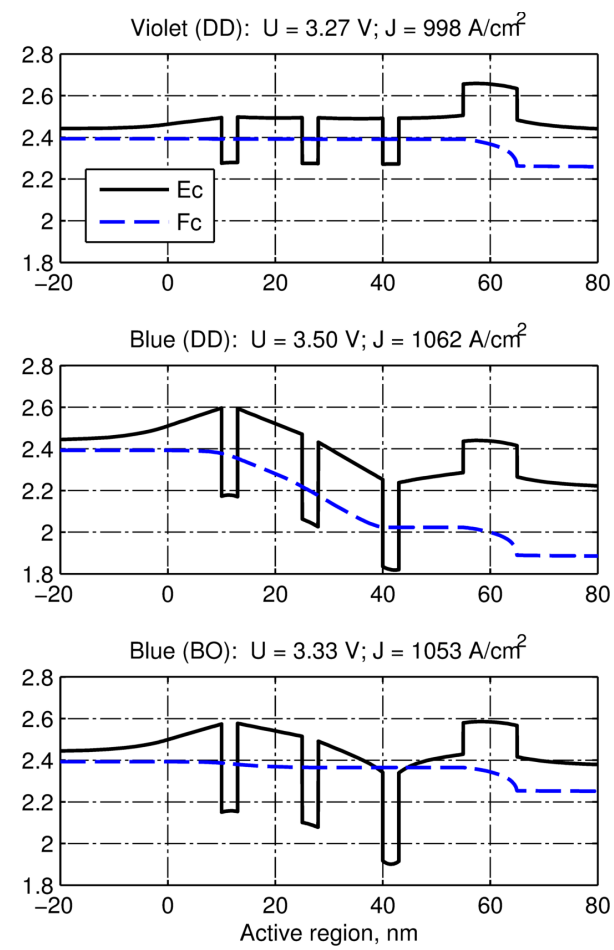

Fig. 1. Conduction band profiles in 3-QW active regions of viole- and blue-emitting LEDs at comparable level of injection curent calculated in drift-diffusion model (DD, two upper panels) and with account of QW ballistic oversoot (BO, lower panel).

\section{INTRODUCTION}

Visible light emitters with enhanced efficiency require MQW active region design. Inhomogeneous carrier injection across the MQW active region imposes unequal and imbalanced QW populations and critically limits the device performance $[1,2]$. In light-emitting diodes (LEDs), the overpumped QWs reduce the overall device efficiency due to the droop phenomena. In laser diodes (LDs), the underpumped QWs directly contribute to optical loss.

In this work, we compare LEDs of different emission ranges, violet, blue, and green, characterized by progressively deeper QWs. To simplify comparison, all structures are considered nonpolar with the same 3-QW layout. For simulations we use MATLAB/COMSOL based Optoelectronic Device Modeling Software (ODMS) developed at Ostendo Technologies Inc. [3].

\section{Simulation RESUlTS}

Figure 1 shows conduction band profiles in violet- and blue-emitting structures at comparable injection currents. Blue LED is characterized by deeper QWs and demonstrates strong electrical inhomogeneity of the active region which notably decreases when electron ballistic overshoot of QW layers is taken into consideration.
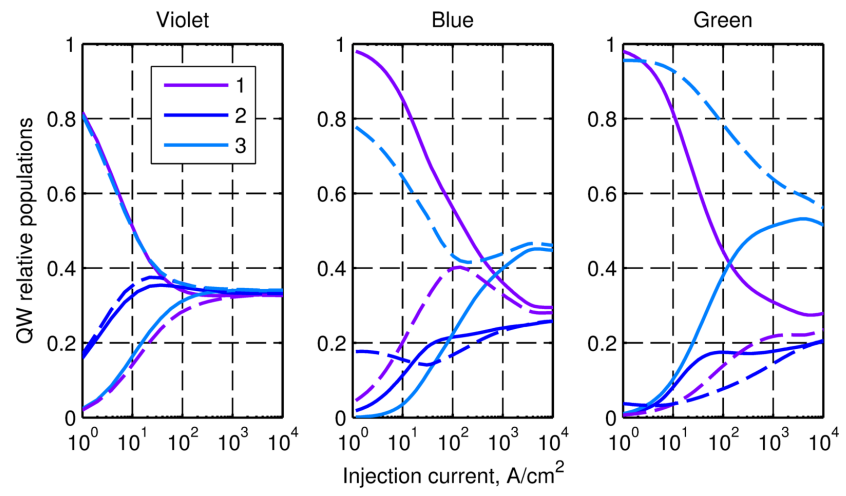

Fig. 2. Relative populations of active QWs in 3-QW LEDs of different emission ranges. QWs are counted from N-side of the diode. Solid lines indicate electron, dashed lines - hole populations. The same QW carier capture times were assumed for all structures: electrons $20 \mathrm{fs}$, holes $10 \mathrm{fs}$. 

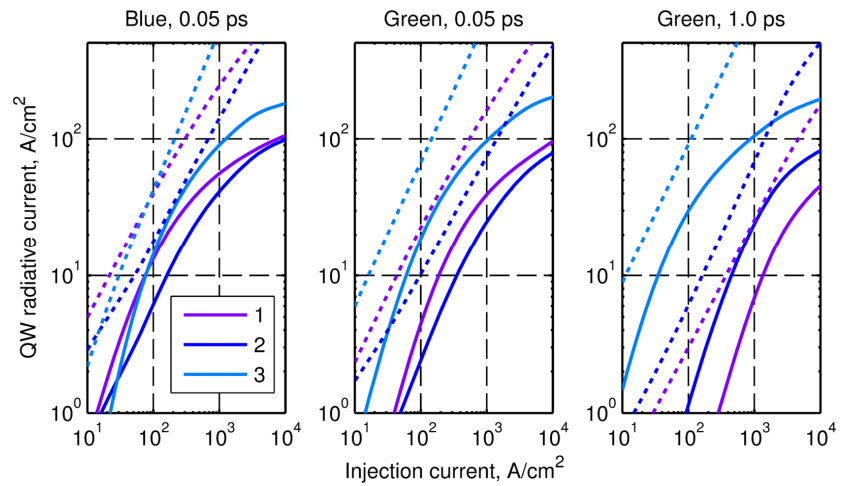

Fig. 3. QW radiative currents in 3-QW blue- and green-emitting LEDs with the same QW carrier capture time (left and middle panels). Dotted lines indicate the total QW currents. Right panel shows the effect of lower QW capture rate with electron capture time of 1 ps.

Figure 2 illustrates the importance of active QW depths in developing the inhomogeneous injection process. At the earlier stage of injection, populations of marginal QWs, \#1 (N-side) and \#3 (P-side), are highly imbalanced in all structures, however, only in LED with deepest QWs (green), the residual QW charges remain large at the highest level of injection. For all QWs, carrier capture times were assumed the same and taken in tens of femtosecond range [4].

Figure 3 shows the effect of carrier capture time variation in drift-diffusion model. Two left panels demonstrate stronger inhomogeneity of QW optical output (in terms of radiative current) in green-emitting structure. Lower rate of electron capture, i.e. longer capture time, promotes the electron transport through the active region but, surprisingly, results in even more unequal QW output. This is the direct consequence of the large DOS capacity of deep hole QWs in green-emitting LED, which allow also for higher electron accumulation in the P-side QW preceding the electron blocking layer.

The same trend can be readily observed when ballistic overshoot is taken into account. Again, electron transport through the active region is enhanced and strong electron accumulation on the diode P-side is indeed observed in simulation; see also Figure 1, lower panel.
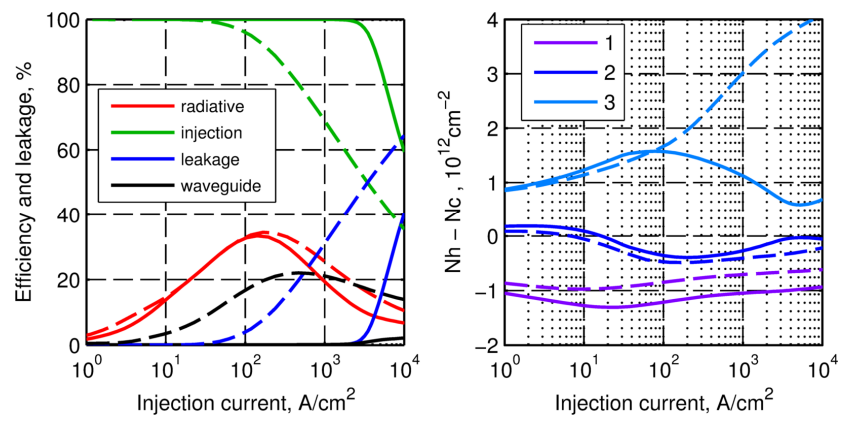

Fig. 4. Left panel: blue-LED radiative and injection efficiencies, electron leakage, and waveguide recombination losses calculated in drift-diffusion model (solid lines) and with QW ballistic oversoot (dashed lines). Right panel shows the dynamics of corresponding QW residual charges.

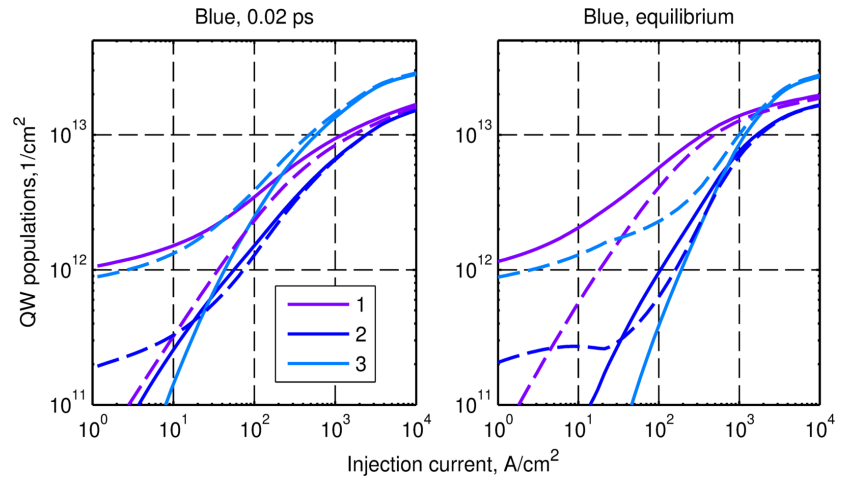

Fig. 5. QW populations in 3-QW blue LED: solid lines - electrons, dashed lines - holes. Left panel: calculated with electron and hole capture times of 20 fs and 10 fs. Right panel: assuming thermodynamical equilibrium with corresponding drift-diffusion carrier subsytem.

Figure 4 illustrates detrimental effect of QW overshooting on the LED efficiency characteristics, carrier leakage, and waveguide recombination losses. Enhanced electron transport triggers earlier start of the electron leakage and deteriorates the total injection efficiency of the structure. Waveguide recombination loss also increases due to large carrier accumulation in front of the electron blocking layer. Similar to lower QW capture rate, the overshooting also leads to dramatic increase of the P-side QW residual charge (right panel).

QW populations in deep optically active QWs are strongly nonequilibrium and crucially depend on the QW carrier capture rates [2]. Figure 5 clarifies the extent of the effect in blueemitting LED structure. Apparently, nonequilibrium QW populations, limited by final $\mathrm{QW}$ capture rates, are lower and more balanced than populations induced by thermodynamic equilibrium with corresponding mobile carrier subsystem.

\section{CONCLUSIONS}

Inhomogeneous QW injection in MQW light-emitting devices is one of the main factors impeding the efficiency of light emitters in the visible spectral range. Besides strong disparity of electron and hole transport in III-nitride materials, the excessive depth of active QWs is equally responsible for the active region electrical non-uniformity and uneven QW injection in GaN-based light emitters.

\section{REFERENCES}

[1] M. V. Kisin and H. S. El-Ghoroury, "Modeling of injection characteristics of polar and nonpolar III-nitride multiple quantum well structures," Journal of Applied Physics, vol. 107, pp. 103106-9, 2010.

[2] M. V. Kisin, C.-L. Chuang, and H. S. El-Ghoroury, "Non-equilibrium QW populations and active region inhomogeneity in polar and nonpolar III-nitride light emitters," Journal of Applied Physics, vol. 111, pp. 103113-9, 2012

[3] M.V. Kisin and H. S. El-Ghoroury, "Modeling of III-Nitride Multiple Quantum Well Light Emitting Structures", IEEE Journal of Selected Topics in Quantum Electronics, vol. 19(5), September 2013, in press.

[4] S. Dasgupta, J. Lu, Nidhi, A. Raman, C. Hurni, G. Gupta, J. S. Speck, and U. K. Mishra, "Estimation of Hot Electron Relaxation Time in GaN Using Hot Electron Transistors," Applied Physics Express, vol. 6, pp. 034002-4, 2013 